\title{
Parasites: where, why and whence?
}

\author{
SARAH E. RANDOLPH* \\ Department of Zoology, University of Oxford, Oxford, UK
}

As biologists, and specifically parasitologists, we are fortunate to live in an age of complementarity, collaboration and integration alongside competition. No longer do biologists stand at either end of the continuum of analytical scales, from the molecular to the ecological, glowering at each other and arguing for the relative merits of one over the other, as was common for many decades after the dawn of molecular biology (Judson, 1996). Our lucky students have inherited a discipline in which the tools of molecular analysis are regularly used to answer questions on complex organisational and global spatial scales, taking this significant advance for granted without knowing anything different. At the same time, new analytical tools developed principally by ecologists are available to help reconstruct and explain the evolutionary information carried by genetic data.

Such exercises have an extra urgency when applied to parasites (which I use here to include pathogens) whose distributions have obvious public health implications. However sophisticated any novel control device may be, deciding where it should be deployed with maximum efficacy and efficiency depends on knowing the parasites' distributions and prevalence levels (i.e. the relative need for intervention) and the relative fragility of their transmission systems (i.e. the likely ease of control). Not only do these two indicators for effective intervention rarely coincide, they are also not static. As the papers in this special issue make clear, we humans are commonly the architects, as well as the victims, of dynamic changes in parasite distributions. All the more reason for parasitologists to develop improved tools to monitor, describe, explain, predict and thereby control the ever-changing threat of infectious diseases.

We start with one of the modern drivers of epidemiology, the aeroplane as a principal means of parasite movement, allowing more or less instantaneous dispersal over vast distances (Tatem et al. 2012). Given the speed and distance of such

* For correspondence: Professor Sarah E. Randolph, Department of Zoology, South Parks Road, Oxford, OX1 3PS, UK. E-mail: sarah.randolph@zoo.ox.ac.uk; Tel: 01865 271241. Fax: 01865271240 transport, it is essential that new epidemiological phenomena are monitored and co-ordinated in real time, no matter how remote the events, for which modern electronic communication tools are ideal, but only if the emergent signals are subject to careful quality control and interpretation (Chunara et al. 2012; Madder et al. 2012). Notwithstanding everimproving and increasingly ubiquitous electronic wizardry, its presence depends on human presence, which is not the case for all pathogens that have the potential to become zoonotic, or even eventually human-transmitted, if the distributions of wildlife and humans change and start to intersect.

Where should we look to be on guard against emergent diseases? Over the past two decades, predictive risk mapping has emerged as a powerful tool, empowered by the parallel growth of information on the environment available at appropriate spatial and temporal scales from satellite imagery. The primary aim may be simply to provide blueprints to help direct surveillance and control programmes, but the best analyses can yield the additional bonus of a deeper understanding of why organisms are found where they are. This can be deduced from identifying the precise predictors of each distribution, and relating them to the underlying biology of transmission. It is easy to produce descriptive maps using off-the-shelf software, but very much more demanding to handle spatiallyexplicit data appropriately to achieve accurate and meaningful predictive maps, filling in the gaps in our knowledge. Spatial auto-correlations and crosscorrelations may be exploited to improve these predictions using kriging techniques derived from the discipline of mining research, although such approaches are not designed to identify biologically important driving variables (Rogers and Sedda, 2012). Interestingly, Rogers and Sedda emphasize that, in contrast to other statistical methods (Rogers, 2006), the techniques of kriging and co-kriging, although increasingly widely used by biologists, cannot in fact throw light on the biological processes, i.e. the why and whence of species distributions, but only on the where, which is, after all, the principal interest of mineral exploration and exploitation. Nevertheless, geo-statistical techniques can be developed within a Bayesian framework to allow us to 
answer interesting biological questions by extracting the maximum amount of useful information from commonly limited data sets comprising continuous spatial variation or discrete spatial variation or spatial point processes (Pullan et al. 2012). Whilst extrinsic environmental correlates of distributional patterns may be captured by these spatial methods, additional effects operating within host populations, especially the complex and geographically variable behaviour in human societies, require a different set of tools.

Fundamentally, parasites differ little from freeliving organisms in that their distributions are all governed by the rates of demographic processes, the building blocks of ecology, with populations surviving only where birth (and immigration) rates on average exceed death (and emigration) rates. Differential abundance is due to variation in space and time in the balance of these rates, along with the additional essential feature of parasites, their specific routes of transmission. The integration of these fundamental concepts of population ecology into process-based epidemiology allows the causes of observed epidemic patterns to be dissected. While this has obvious relevance to public health, advances in understanding in the opposite direction, from epidemiology to theoretical ecology, is potentially offered by the rapid and often cyclic dynamics characteristic of micro-parasites and the large accessible datasets characteristic of human hosts (patients). Analytical techniques have become increasingly sophisticated since the basis of this crucial insight was first published (Anderson and May, 1979; May and Anderson, 1979), particularly with respect to travelling waves of epidemics, applied first to measles across the UK (Grenfell et al. 2001) and here to pertussis in Thailand (Blackwood et al. 2012). It is clear that immunisation programmes must take account of geographically distinct and changing human demographic patterns to be maximally effective.

The rapid rates of genetic change characteristic of small organisms, not to mention viruses of all sorts and RNA viruses in particular, introduce variability in host-parasite interactions and permit differential persistence of genetically distinct viruses across heterogeneous landscapes. Thus genetic information must be added to the landscape epidemiology of the above studies. Modern sequence analyses generate ever more robust and credible phylogenetic trees that can be placed in both temporal and spatial contexts, from which the long-term and recent history of each viral strain can be deduced. Exploitation of the resulting environmental and genetic information requires advanced statistical and mathematical modelling techniques to reveal probable routes of dispersal, depending on the biology of transmission. Domestic dog rabies, for example, turns out to differ in significant respects from wildlife rabies dues to the facilitating impact of anthropogenic factors on dispersal, thereby requiring different control strategies (Brunker et al. 2012). While phylogeographics on its own is rarely more than descriptive, when combined with information on the ecology and agedependent infection history and mortality rates of the host population, explanatory processes can be deduced. The necessary integration of field ecology and genetics typically takes a large team of collaborators with complementary skills. One such relatively rare study has revealed considerable detail about the arrival, covert local circulation and outbreak of highly pathogenic avian influenza in wild swans in the UK (Pybus et al. 2012). This study exemplifies the gradual integration of molecular approaches into outbreak investigation and control, driven in part by the collapsing cost of sequencing. Bird migration is the easy scapegoat for the natural movement of parasites round the world, but in this case it is clearly biologically appropriate, backed by accurate knowledge of species-specific ecology and the timing and routes of migration that fit well with the epidemiological events.

In contrast, in my own field of interest concerning tick-borne encephalitis virus (TBEV) in Eurasia, birds are unlikely vehicles because, amongst other contradictory factors, they are not competent reservoir hosts and their migration routes do not match viral dispersal events. Instead, knowledge of human history seems to hold the key. A recent explanation for the anomalous foci of the Far-Eastern subtype of TBEV in the European region of the former Soviet Union, thousand of kilometres west of their recognised endemic region but contained within the western borders of the former Soviet Union, has convincingly pointed the finger at human activity rather than the natural migration of mammals or birds. Specifically, the large-scale predominantly westward redistribution of hundreds of thousands of game animals for economic purposes, which occurred only between the early 1930s and 1974, fits the spatial and temporal patterns of viral introductions deduced from phylogeographic analysis (Kovalev et al. 2010). Similarly, the westward dispersal of the Siberian subtype of TBEV in the Middle Urals and the European part of Russia appears to have originated twice from different foci in western Siberia. The concordance of phylogenetic analysis and history suggests that these events were related to the first land road into Siberia (from Moscow to Narym) about 400 years ago and then to the construction of the Trans-Siberian Way (from Moscow to Irkutsk) about 100 years later (Kovalev et al. 2009). In summary, Kovalev et al. (2009) conclude: "the victims of TBEV in Europe are the price that the European people have paid for the colonization of Siberia". More recently, slow dispersal of the Western subtype of TBEV over the past 50 years within Central Europe (what is now the Czech Republic) is thought to be due to 
anthropogenic factors (Weidmann et al. 2011). Natural geographic and political barriers evidently limited the spread further west until genetically distinct strains of W-TBEV arose about fifteen years ago in small isolated foci in southeast Germany (Weidmann et al. 2011) and have remained geographically highly limited since then (G. Dobler, paper presented at the International Conference on Tick-borne Encephalitis, Irkutsk, Siberia, June 2012), rather than dispersing further as one might expect if birds were the culprit. Low probability random introductions of infected rodents or tickinfested deer (neither of which migrate long distances) into pockets of permissive habitat, possibly but not necessarily through human agency, seem a likely mechanism behind this phylogeographic pattern.

Although the further back one goes in time, the more obscure become the events that have shaped the present, nevertheless phylogeography is proving to be a powerful tool in the study of the where, why and whence of parasite distributions even in relatively deep time (Morand, 2012). The final four papers in this special issue emphasize the potential of this approach to address a wide range of parasitological questions, including host-parasite co-evolution and the implications for genetic control of evidence of invasions by pests from multiple sources (Morgan et al. 2012), the 'One World, One Health' concept that unifies humans, livestock and wildlife as harbingers of shared parasites (Gray and Salemi, 2012), the importance of considering horizontal gene transfer even amongst supposedly clonal bacteria (Margos et al. 2012), and the building of human parasite communities through successive world events from pre-history to the present, "out of Africa”, domestication and finally globalisation (Morand, 2012).

What becomes obvious is the significant role of humans in dispersing parasites around the world over many centuries, even millennia. Although the driving forces for emergence of infectious diseases, anthropogenic or otherwise, have always occurred, nevertheless we may be justified in the current preoccupation with recent events as if they were novel, because those drivers have recently become much more forceful with the acceleration of global trade and travel, which is where we started (Tatem et al., 2012). We are evidently living in a world that is quantitatively, if not qualitatively, different from that of our ancestors (Markov et al., 2012). Fortunately, technological advances (including whole genome sequencing, now at the drop of a hat) and analytical methods are just about keeping up with the need to handle vast arrays of data from a wide variety of sources on all scales of space and time.

\section{ACKNOWLEDGEMENTS}

I am very grateful to all the authors and reviewers who have contributed so positively to this special issue. I now appreciate better the huge task of being a journal editor, for which I sincerely thank Les Chappell with whom it has been a pleasure to work. David Rogers and Oliver Pybus kept me on my toes with this preface.

\section{REFERENCES}

Anderson, R. M. and May, R. M. (1979). Population biology of infectious diseases: Part 1. Nature 280, 361-367.

Blackwood, J. C., Cummings, D. A. T., Broutin, H., Iamsirithaworn, S. and Rohani, P. (2012). The population ecology of infectious diseases: pertussis in Thailand as a case study. Parasitology 139, 1888-1898.

Brunker, K., Hampson, K., Horton, D. L. and Biek, R. (2012). Integrating the landscape epidemiology and genetics of RNA viruses: rabies in domestic dogs as a model. Parasitology 139, 1899-1913.

Chunara, R., Freifeld, C. C. and Brownstein, J. S. (2012). New technologies for reporting real-time emergent infections. Parasitology 139, 18431851.

Gray, R. R. and Salemi, M. (2012). Integrative molecular phylogeography in the context of infectious diseases on the human-animal interface. Parasitology 139, 1939-1951.

Grenfell, B. T., Bjornstadt, O. N. and Kappey, J. (2001). Travelling waves and spatial hierarchies in measles epidemics. Nature 414, 716-723.

Judson, H. F. (1996). The Eighth Day of Creation: Makers of the Revolution in Biology. Cold Spring Harbor Laboratory Press, New York.

Kovalev, S. Y., Chernykh, D. N., Kokorev, V.S., Snitkovskaya, T.E. and Romanenko, V.V. (2009). Origin and distribution of tick-borne encephalitis virus strains of the Siberian subtype in the Middle Urals, the north-west of Russia and the Baltic countries. Fournal of General Virology 90, 2884-2892.

Kovalev, S. Y., Kokorev, V. S. and Belyaeva, I. V. (2010). Distribution of Far-Eastern tick-borne encephalitis virus subtype strains in the former Soviet Union. Fournal of General Virology 91, 2941-2946.

Madder, M., Walker, J. G., Van Rooyen, J., Knobel, D., Vandamme, E., Berkvens, D., Vanwambeke, S. O. and De Clercq, E. M. (2012). e-Surveillance in Animal Health: use and evaluation of mobile tools. Parasitology 139, 1831-1842.

Margos, G., Castillo-Ramirez, S. and Gatewood-Hoen, A. (2012). Phylogeography of Lyme borreliosis-group spirochetes and methicillinresistant Staphylococcus aureus. Parasitology 139, 1952-1965.

Markov, P. V., van de Laar, T. J., Thomas, X. V., Aronson, S. J., Weegink, C.J., van den Berk, G. E., Prins, M., Pybus, O. G. and Schinkel, J. (2012). Colonial history and contemporary transmission shape the genetic diversity of Hepatitis C virus genotype 2 in Amsterdam. Fournal of Virology 86, 7677-7687.

May, R. M. and Anderson, R. M. (1979). Population biology of infectious diseases: part 2. Nature 280, 455-461.

Morand, S. (2012). Phylogeography helps with investigating the building of human parasite communities. Parasitology 139, 1966-1974.

Morgan, E. R., Clare, E. L., Jefferies, R. and Stevens, J. R. (2012). Parasite epidemiology in a changing world: can molecular phylogeography help us tell the wood from the trees? Parasitology 139, 1924-1938.

Pullan, R. L., Sturrock, H. J. W., Soares Magalhaes, R. J., Clements, A. C. A. and Brooker, S. J. (2012). Spatial parasite ecology and epidemiology: a review of methods and applications. Parasitology 139, 1870-1887.

Pybus, O. G., Perrin, C. M., Choudhury, B., Manvell, R. J., Nunez, A., Schulenburg, B., Sheldon, B. C. and Brown, I. H. (2012). The ecology and age structure of a highly pathogenic avian influenza virus outbreak in wild mute swans. Parasitology 139, 1914-1923.

Rogers, D. J. (2006). Models for vectors and vector-borne diseases. Advances in Parasitology 62, 1-37.

Rogers, D. J. and Sedda, L. (2012). Statistical models for spatially explicit biological data. Parasitology 139, 1852-1869.

Tatem, A. J., Huang, Z., Das, A., Qi, Q., Roth, J. and Qiu, Y. (2012). Air travel and vector-borne disease movement. Parasitology 139, 1816-1830.

Weidmann, M., Ruzek, D., Krinanec, K., Zoller, G., Essbauer, S., Pfeffer, M., Zanotto, P. M. D. A., Hufert, F. T. and Dobler, G. (2011). Relation of genetic phylogeny and geographical distance of tick-borne encephalitis virus in central Europe. Fournal of General Virology 92, 19061916. 